\title{
Focused-Ion-Beam Direct-Writing of Ultra-Thin Superconducting Tungsten Composite Films
}

\author{
Wuxia Li, J. C. Fenton, and P. A. Warburton
}

\begin{abstract}
This article was published as IEEE Trans. Appl. Supercond. (2009) 192819 (http://dx.doi.org/10.1109/TASC.2009.2019251) (C) 2009 IEEE. Personal use of this material is permitted. Permission from IEEE must be obtained for all other uses, in any current or future media, including reprinting/republishing this material for advertising or promotional purposes, creating new collective works, for resale or redistribution to servers or lists, or reuse of any copyrighted component of this work in other works.
\end{abstract}

\begin{abstract}
Tungsten composite films of thickness as low as $19 \mathrm{~nm}$ have been deposited using a $30 \mathrm{keV}^{\mathrm{Ga}} \mathrm{C}^{+}$focused-ion-beam with tungsten carboxyl $\left(\mathrm{W}(\mathrm{CO})_{6}\right)$ as the gas precursor. Films of thickness $25 \mathrm{~nm}$ or more are superconducting with a transition temperature exceeding $5 \mathrm{~K}$. Films in the thickness range $25 \mathrm{~nm}$ to $50 \mathrm{~nm}$ show an increasing $T_{c}$ for a decreasing film thickness. This correlates well with the measured dependence of the normal state resistivity upon film thickness. We attribute this behavior to an increase in the BCS electron-phonon interaction potential resulting from a reduction in the electron mean-free-path as the film thickness is reduced. In the light of these data we discuss the applicability of FIB-deposited tungsten for devices requiring ultra-thin superconducting films, including photon detectors and phase-slip qubits.
\end{abstract}

Index Terms - Direct-writing, Focused-ion-beam, Superconductivity, Tungsten Composite, Thin Film

\section{INTRODUCTION}

$\mathrm{F}$

or the future of nanotechnology, the controlled growth and manipulation of materials at the nanoscale are very important, and in particular, site-specific direct assembly techniques for critical level nanoscale processing. Recently, the use of a focused ion beam to induce the deposition of materials from metal-organic precursors has emerged as an important technique for growing nanomaterials to form complex functional units in a single "writing" step [1], [2].

Highly disordered transition metals generally display a higher $T_{c}$ than their annealed ordered counterparts. Pure single-crystal bcc $\mathrm{W}$ has a very low $T_{c}(\sim 15 \mathrm{mK})$. Disordered or granular $\mathrm{W}$ films alloys show significant $T_{c}$ enhancements [3]. The superconductivity of FIB-deposited tungsten was first demonstrated by Sadki et al. [4] then by Luxmoore et al. [5]. Both groups reported a $T_{c}$ of $5.2 \mathrm{~K}$ for such material and qualitatively ascribe the comparatively high $T_{c}$ to the absence of long-range order in the material crystal structure. In our previous work, systematic experiments were carried out to quantitatively confirm that the enhanced $T_{c}$ in FIB-deposited tungsten is directly related to how close the deposited material lies to the metal-insulator transition (MIT). By varying the ion-beam deposition current, the proximity to the MIT can be controlled within a certain range. We also investigated the controlled growth and superconductivity of "flying" three-dimensional W-containing nanostructures [6].

The effect of ion dose on the growth and room temperature resistivity of FIB deposited tungsten composites has previously been studied [7], [8]. However, up to now an investigation of the superconductivity of ultra-thin tungsten composite films grown by this method is still lacking. Here we report the flexible and controllable growth and temperature dependent electrical properties of tungsten composite thin films. In particular, the evolution of the growth morphology, growth rate and the thickness-dependent superconducting properties are presented. The possible applications of such materials include fabrication of superconducting single photon detectors (SSPD's) [9] and qubits based on quantum phase slip centers (QPSC's) [10].

\section{EXPERIMENTAL}

A commercially available dual beam scanning electron microscope (SEM)/focused-ion-beam (FIB) system supplied by Carl Zeiss was used for tungsten composite deposition utilizing a beam of $30 \mathrm{keV}$ singly charged $\mathrm{Ga}^{+}$ions. Before the tungsten deposition, gold contact patterns were formed onto the oxidised silicon substrates by conventional photolithography-based processes [6]. For site-specific tungsten deposition, $\mathrm{W}(\mathrm{CO})_{6}$ gas was injected onto the sample surface through a nozzle, creating a local high pressure in the region scanned by the ion beam. The base pressure before introducing the precursor gas was $8.3 \times 10^{-6}$ mbar and the pressure was in the range of $1.1-1.3 \times 10^{-5}$ mbar during deposition.

The four-terminal configurations were fabricated by the following steps: first, the tungsten strip under test was grown with a 20

This work was supported by the Engineering and Physical Sciences Research Council (EPSRC), United Kingdom, grant reference EP/F035411/1.

Wuxia Li, J. C. Fenton, and P. A. Warburton are with the London Centre for Nanotechnology, University College London, 17-19 Gordon Street, London, WC1H OAH, United Kingdom. 
$\mathrm{pA}$ ion-beam current scanned over a $20 \mu \mathrm{m} \times 1 \mu \mathrm{m}$ rectangular area; then 4 other thicker strips were deposited using identical deposition conditions to connect the strip of interest to the large Au pads. The ion beam is scanned rapidly in one direction and slowly in the perpendicular direction. Specifically the scan frequencies are $20,000 \mathrm{~Hz}$ and $0.02 \mathrm{~Hz}$ in the X and Y directions respectively, where the $\mathrm{X}$ direction lies along the length of the nanowire and the $\mathrm{Y}$ direction is transverse to it. Thus the whole area to be deposited is scanned in a time of $50 \mathrm{~s}$; this we refer to as a single "loop scan". In order to retain a constant film thickness across the width of the deposited film we always deposit an integer number, $\mathrm{N}_{\mathrm{s}}$, of loop scans, with total deposition time $50 \mathrm{~N}_{\mathrm{s}}$ seconds. At the deposition current of $20 \mathrm{pA}$ the ion dose per loop scan is $0.05 \mathrm{nC} / \mu \mathrm{m}^{2}$.

The growth, scanning loop by scanning loop, was monitored by in-situ SEM imaging. The width of the strip was measured by SEM and the thicknesses were measured by a Dektak 8 Surface Profiler and by Atomic Force Microscope. The chemical composition analysis was performed by energy dispersive X-ray spectroscopy (EDS). Features used for EDS were grown with a nominal scan area of $2.0 \mu \mathrm{m} \times 2.0 \mu \mathrm{m}$ and are $1 \mu \mathrm{m}$ thick.

Low-temperature transport properties were obtained using current-biased transport measurements. The sample was mounted on a "dip-stick", which was immersed in a liquid-helium storage dewar. The chip under test was first stuck to a copper chip-carrier by silver paste and dried in air, and then gold wire bonding was used to connect the gold contact pads on the chip to copper pads on the chip carrier. The chip carrier was mounted on a copper block at the end of the dip-stick probe. The temperature was measured by a Lakeshore Cernox resistance temperature sensor; this was attached to the copper block and connected to an Oxford Instruments Intelligent Temperature Controller. Transport measurements were performed with a home-made current source and voltage amplifier. All the data acquisition and analysis were controlled by a LabVIEW-based platform.

\section{RESULTS AND DISCUSSION}

Focused-ion-beam induced tungsten deposition occurs when $\mathrm{W}(\mathrm{CO})_{6}$ molecules are decomposed during ion beam exposure. W, $\mathrm{C}$ and a small amount of $\mathrm{O}$ are then absorbed to the substrate surface to form the nonvolatile deposits. The deposit also contains $\mathrm{Ga}$ from the ion source. Energy dispersive X-ray spectroscopy (EDS) analysis shows that the deposit grown using a $20 \mathrm{pA}$ ion beam current has 53 at $\%, 34$ at $\%, 11$ at $\%$ and 2 at $\%$ of $\mathrm{C}, \mathrm{W}, \mathrm{Ga}$ and $\mathrm{O}$ respectively.

Figure 1 shows the evolution of the surface topography of tungsten deposits grown after various numbers $\mathrm{N}_{\mathrm{s}}$ of loop-scans. The corresponding images reveal that for $\mathrm{N}_{\mathrm{s}}=1$, isolated nanoscale islands nucleate, scattered over the beam scanning area. Due to the isolated nature, these samples are open circuit. For $\mathrm{N}_{\mathrm{s}}=2$, these islands elongate and merge to form networks. With $\mathrm{N}_{\mathrm{s}}=3$, material deposited becomes contiguous though porous and with discernable uneven thickness distribution. For $\mathrm{N}_{\mathrm{s}}=4$, the coalescence of the networks continues and increases the coverage of the area scanned by the beam. After exposure up to $\mathrm{N}_{\mathrm{s}}=6$, the network structures merge to a continuous solely planar surface and further growth becomes homogeneous $\left(\mathrm{N}_{\mathrm{s}}=8\right)$. Similar observation has been previously reported on the ion dose dependent growth of tungsten-containing materials by FIB [8]. High-resolution transmission electron microscope (HRTEM) measurements show that the tungsten-containing films grown by FIB do not display any long-range order [5], [6].

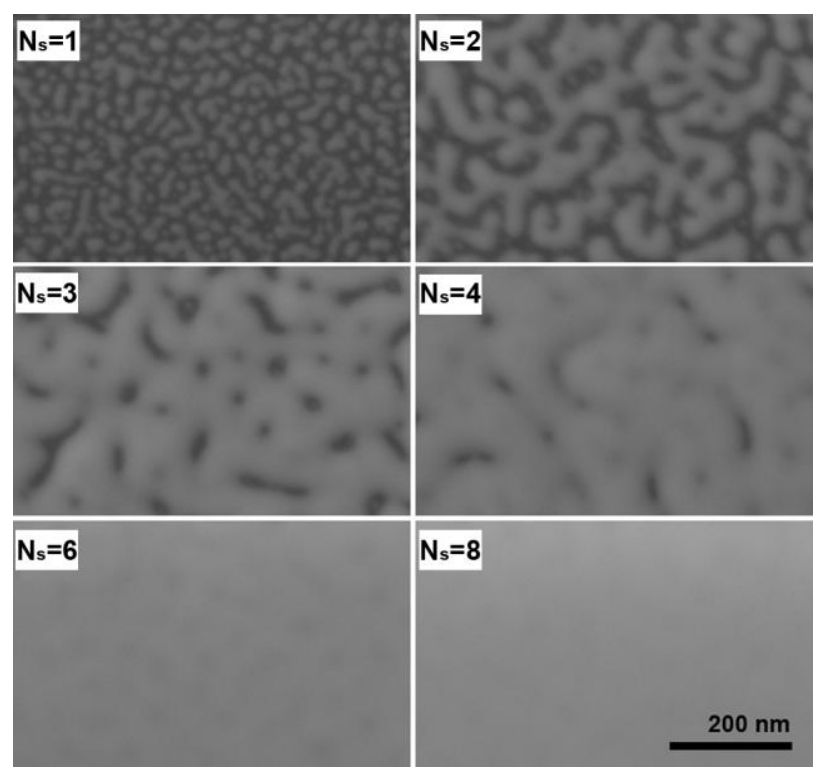

Fig.1 SEM plan-view images of tungsten composites grown using a $20 \mathrm{pA}$ ion-beam current. $\mathrm{N}_{\mathrm{s}}$ is the number of loop scans, where the total ion dose is $0.05 \mathrm{~N}_{\mathrm{s}} \mathrm{nC} / \mu \mathrm{m}^{2}$. 
The dependence of the thickness on $\mathrm{N}_{\mathrm{s}}$ is shown in Fig.2. The growth rate actually is affected by many factors, such as substrate material, ion beam current density, beam scan strategy, the precursor molecule supply rate and substrate temperature during growth [2], [3], [6]-[8]. By changing $\mathrm{N}_{\mathrm{s}}$ under otherwise identical condition, homogeneous and linear growth with a rate of $3.2 \mathrm{~nm}$ per loop-scan has been observed for films grown with $\mathrm{N}_{\mathrm{s}}>6$.

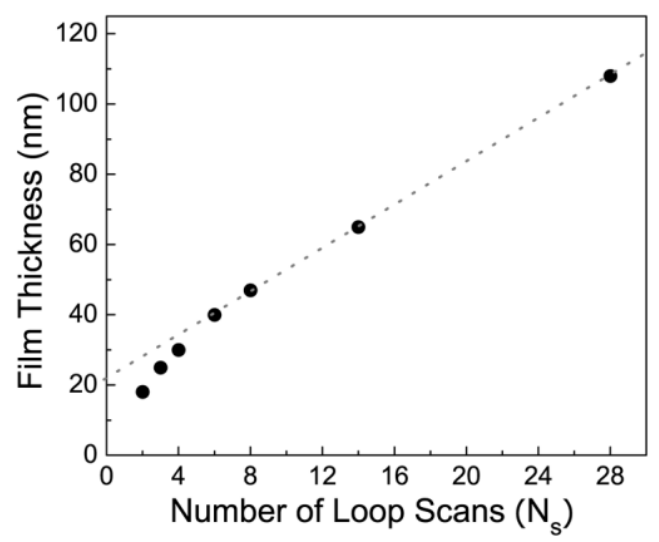

Fig. 2 Average film thickness $v s \mathrm{~N}_{\mathrm{s}}$ for tungsten composites grown using a $20 \mathrm{pA}$ ion-beam current. The dotted line is the linear fit to the data for films grown with $\mathrm{N}_{\mathrm{s}}>6$.

A typical four-terminal configuration for a measurement of the temperature dependence of the resistivity is shown in Fig.3. Here the strip under test was deposited using $\mathrm{N}_{\mathrm{s}}=3$. The distance between the two inner tungsten electrodes is $10 \mu \mathrm{m}$, a distance large enough to ensure that there is no shorting across the sample when the four wiring connections are deposited. The image taken under a higher magnification of the selected area (white rectangle) reveals that after deposition of a thicker tungsten strip on top, the network structure is preserved.

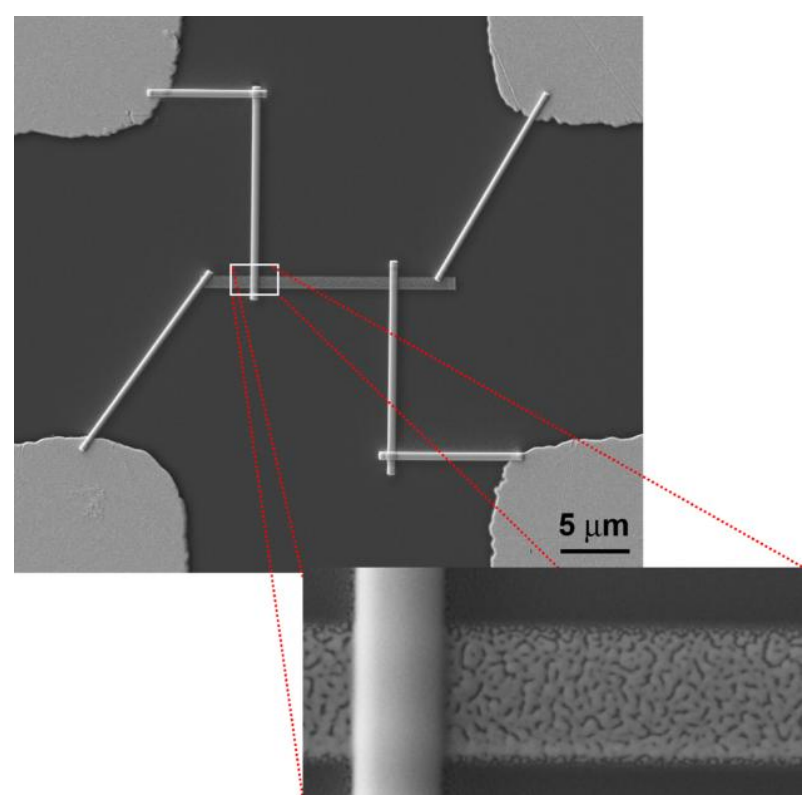

Fig. 3 SEM image of a four-terminal configuration for a temperature dependent resistivity measurement with $\mathrm{N}_{\mathrm{s}}=3$. The white rectangle is also shown at a higher magnification.

Fig. 4 (a) shows the resistivity as a function of temperature for films grown with varying $\mathrm{N}_{\mathrm{s}}$. For $\mathrm{N}_{\mathrm{s}}=1$ and 2 , since physically the nanoscale islands are isolated and the networks are non-continuous, these samples are electrically insulating. For films grown with $\mathrm{N}_{\mathrm{s}} \geq 3$ the normal state resistivity is very weakly temperature dependent, changing by less than $5 \%$ from room temperature to $7 \mathrm{~K}$. This indicates that FIB deposited tungsten lies close to the metal-insulating transition [6]. Fig. 4 (b) shows a magnified view of the low-temperature region. All films with $\mathrm{N}_{\mathrm{s}} \geq 3$ are superconducting with a $\mathrm{T}_{\mathrm{c}}$ exceeding $5 \mathrm{~K}$. 

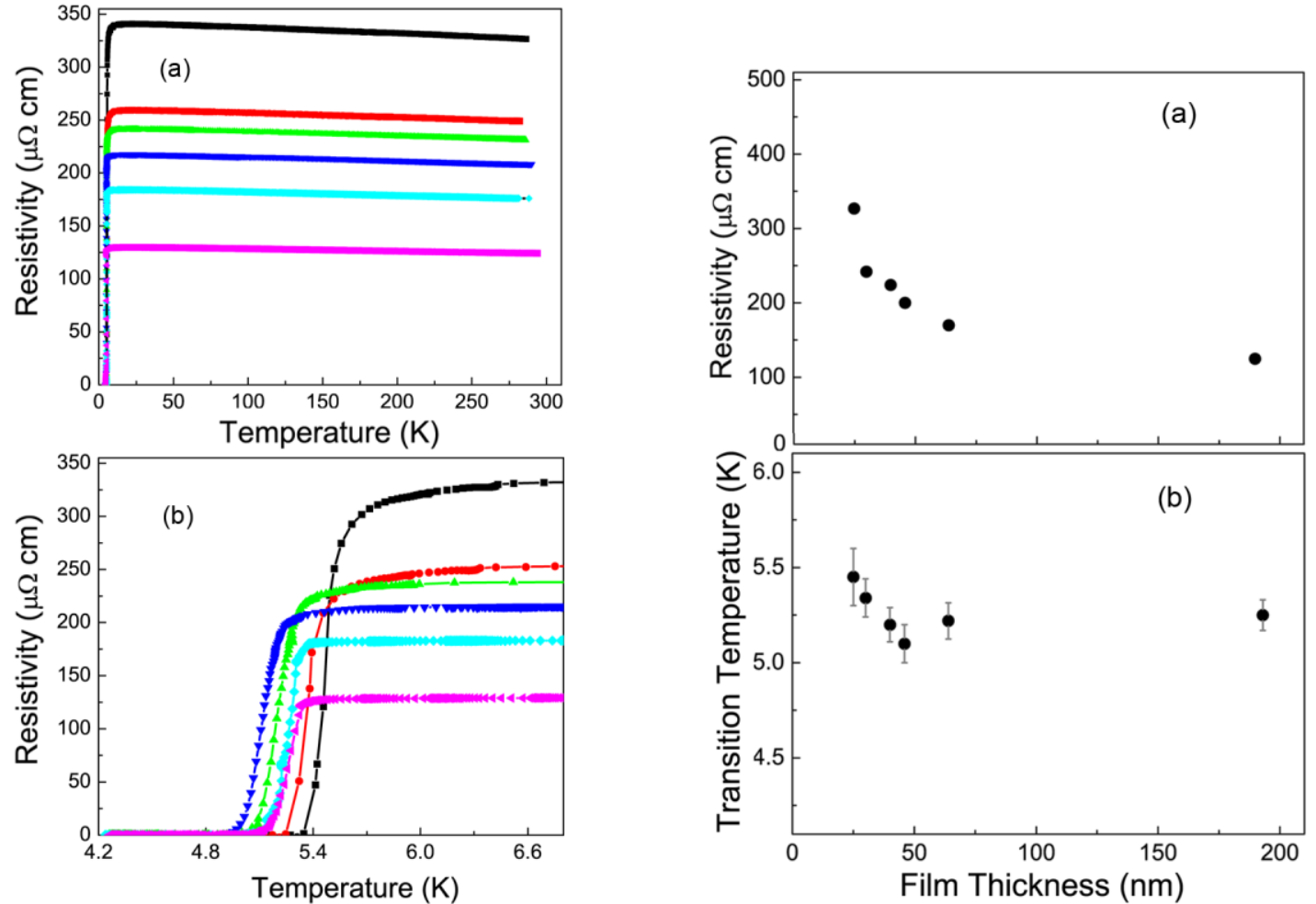

Fig. 4 (left) Temperature dependence of the resistivity of the tungsten films: (a) $\rho(T)$ for films grown with $\mathrm{N}_{\mathrm{s}}$ of 3, 4, 6, 8, 11 and 52 (from top to bottom) respectively; (b) shows the superconducting transitions in the low-temperature region. The lines are guides to the eye.

Fig. 5 (right) Resistivity (a) and transition temperature (b) vs film thickness for films grown with a $20 \mathrm{pA}$ ion-beam current.

The dependence of the room-temperature resistivity upon the film thickness is shown in Fig. 5(a). The resistivity of our samples initially decreases with increasing thickness before reaching a constant value of between 100 and $150 \mu \Omega \mathrm{cm}$ for films thicker than about $50 \mathrm{~nm}$ (see also [2]). Qualitatively similar results have previously been reported in investigations of the ion-dose-correlated normal-state resistivity of FIB deposited tungsten by Takahashi et al. [7]. In the low ion-dose region $\left(0.08-0.096 \mathrm{nC} / \mu \mathrm{m}^{2}\right)$, the resistivity rapidly decreases with increasing ion dose; at higher doses $\left(>0.112 \mathrm{nC} / \mu \mathrm{m}^{2}\right)$, it is constant. (In our experiments, however, the transition from the low-dose region to the high-dose region occurs around $\mathrm{N}_{\mathrm{s}}=8$, i.e. at a dose of $0.4 \mathrm{nC} / \mu \mathrm{m}^{2}$.) Takahashi et al. ascribed the higher resistivity at low ion-dose to an overestimate of the cross-section of the films. Apart from this, we believe another possible reason for this phenomenon is that for ultra-thin films, the electron mean free path against elastic scattering is mainly determined by the surface scattering. Thus by reducing the thickness of the ultra-thin films, the electron mean free path decreases below its bulk value, which in turn leads to an increase in resistivity. This model would suggest that the bulk (i.e. defect-limited) electron mean-free-path in FIB-deposited tungsten is around $50 \mathrm{~nm}$.

The dependence of $\mathrm{T}_{\mathrm{c}}$ upon the film thickness for $\mathrm{N}_{\mathrm{s}} \geq 3$ is shown in Fig. 5(b). Here $T_{c}$ is defined as the temperature at which the resistivity falls to $50 \%$ of its value at the onset of the transition. The error bars in Fig. 5(b) correspond to the width of the measured resistive transition, defined by the temperatures at which the resistivity falls to $90 \%$ and $10 \%$ of its value above $\mathrm{T}_{\mathrm{c}}$. The trend for the thickness-dependence of $T_{c}$ is rather similar to that for the normal-state resistivity shown in Fig. 5(a) - $T_{c}$ decreases with increasing thickness up to about $50 \mathrm{~nm}$. We therefore speculate that the enhanced $T_{c}$ in our thinnest films arises due to the reduced electron mean-free-path. Osofksy et al. [11] have previously demonstrated that, near the MIT, reducing the mean-free-path leads to an increase in the electron screening length which in turn leads to an increase in the BCS electron-phonon interaction potential.

Our films have a large critical-current density $\left(J_{c}\right)$ of order of $1.0 \mathrm{MA} / \mathrm{cm}^{2}$ at $4.2 \mathrm{~K}$, indicating that the transport is not weak-link limited. The $J_{c}$ for the network structure deposited with $\mathrm{N}_{\mathrm{s}}=3$ is crudely estimated at $1.74 \mathrm{MA} / \mathrm{cm}^{2}$. This is somewhat larger than that for films with a flat surface; for example, for a film $190 \mathrm{~nm}$ in thickness, the $J_{c}$ is $1.1 \mathrm{MA} / \mathrm{cm}^{2}$. Considering that the cross-section of the network structure is likely to have been overestimated since we evaluated it neglecting the presence of the voids; the intrinsic $J_{c}$ of such a structure is probably somewhat larger still. This may suggest that the voids in the film are acting as vortex pinning sites. 


\section{CONCLUSION}

In conclusion, we are able to grow tungsten composites by focused-ion-beam induced deposition. Nanoscale islands, network-like structures, porous thin films and films with flat surface can be fabricated, which may offer great flexibility to grow nanoscale disordered materials systems to explore the interplay of superconductivity and morphology [12]. Deposits with contiguous structures all have technologically useful $T_{c}$ of above $5 \mathrm{~K}$. Such materials are amorphous and films with very uniform surface can be achieved without grain boundaries. Also using this technique contacts can be made using the same material, avoiding out a number of experimental problems associated with interfaces [13].

In terms of many applications, the key question is how thin a superconducting film can be made. In our work the thinnest films which show a superconducting transition are $25 \mathrm{~nm}$ thick. This is significantly larger than the coherence length of $5.9 \mathrm{~nm}$ for FIB-deposited tungsten as measured by Sadki et al. [4]. Further reductions in film thickness will therefore be required if FIB-deposited tungsten is to be used for superconducting single-photon detectors [14] and/or devices utilising quantum phase-slip centres [10]. Nevertheless, the nanoscale islands shown in figure $1\left(\mathrm{~N}_{\mathrm{s}}=1\right)$ may be intrinsically superconducting, though the non-connectivity of the islands prevents us from performing transport measurements. Future experiments therefore include deposition at a lower ion-beam current so as to allow us to grow continuous films at very low ion-dose.

\section{ACKNOWLEDGMENT}

The authors acknowledge Kevin Lee for technical assistance.

\section{REFERENCES}

[1] W. Li, and T. H. Shen, "Rectifying "nanohomo" contacts of W-Ga-C composite pad and nanowire fabricated by focused-ion-beam-induced chemical vapor deposition", Appl. Phys. Lett. Vol. 87, p.p. 123113, Sept. 2005.

[2] W. Li, and P. Warburton, "low-current focused-ion-beam induced deposition of three-dimensional tungsten nanoscale conductors", Nanotechnology, vol.18, pp. 485305 , Oct. 2007.

[3] S. Kondo, "Superconducting characteristics and the thermal stability of tungsten-based amorphous thin films" J. Mater. Res. Vol. 7, pp. 853-860, Apr. 1992.

[4] E. S. Sadki, S. Ooi, and K. Hirata, "Focused-ion-beam-induced deposition of superconducting nanowires," Appl. Phys. Lett., vol. 85, pp. 6206, Dec. 2004.

[5] I. J. Luxmoore, I. M. Ross, A. G. Gullis, P. W. Fry, J. Orr, P. D. Buckle, and J. H. Jefferson, "Low temperature electrical characterisation of tungsten nano-wires fabricated by electron and ion beam induced chemical vapour deposition", Thin Solid Films, Vol. 515, pp. 6791-6797, Feb. 2007.

[6] W. Li, J. C. Fenton, Y. Wang, D.W. McComb, and P. A. Warburton "Direct-writing and tunability of the superconductivity of three-dimensional tungsten nanostructures grown by focused-ion-beam deposition", Adv. Mat., submitted for publication.

[7] Y. Takahashi, Y. Madokoro, and T. Ishitani, "Growth of Tungsten Film by Focused Ion Beam Induced Deposition”, Japanese Journal of Appl. Phys. Vol.30, pp. L518-L520, Jan. 1991.

[8] H. Langfischer, B. Basnar, H. Hutter, and E. Bertagnolli, "Evolution of tungsten film deposition induced by focused ion beam", J. Vac. Sci. Technol. A, Vol. 20, No. 4, pp.1408-1415, Apr. 2002.

[9] G.. N. Gol'tsman, O. Okunev, G.. Chulkova, A. Lipatov, A. Semenov, K. Smirnov, B. Voronov, A. Dzardanov, C. Williams, and R. Sobolewski, "Picosecond superconducting single-photon optical detector" Appl. Phys. Lett, vol.79, pp.705, Aug. 2001.

[10] J. E. Mooij, and Y. V. Nazarov, "Superconducting nanowires as quantum phase-slip junctions", Nature Physics, Vol. 2, pp. 169-172, Mar. 2006.

[11] M. S. Osofsky, R. J. Soulen, Jr., J. H. Claassen, G. Trotter, H. Kim, and J. S. Horwitz, "new insight into enhanced superconductivity in metals near the metal-insulate transition (MIT)", Phys. Rev. Lett., Vol. 87, pp.197004, Oct. 2001

[12] Y. Dubi, Y. Meir, and Y. Avishai, "Nature of the superconductor-insulator transition in disordered superconductors", Nature, Vol. 449, No. 18, pp.876-880, Oct. 2007.

[13] V. N. Antonov, A. F. Volkov, and H. Takayanagi, "Proximity" effect in the conductance of mesoscopic normal metal-superconductor structures, Europhys. Lett., Vol.38, pp.453-458, Apr. 1997.

[14] A. Bezryadin, C. N. Lau, and M. Tinkham, “Quantum suppression of superconductivity in ultrathin nanowires”, Nature, Vol. 404, pp. 971-974, Apr. 2000. 